\title{
Cleaved Silica Microsphere for Temperature Measurement
}

\author{
A. D. Gomes ${ }^{(}$, B. Silveira ${ }^{(}$, J. Dellith, M. Becker, M. Rothhard, H. Bartelt, and O. Frazão
}

\begin{abstract}
A sensing structure based on a cleaved silica microsphere is proposed for temperature sensing. The microsphere was cleaved using focused ion beam milling. The asymmetry in the structure introduced by the cut generates not only new cavities but also random interferometric reflections inside the microsphere. These two spectral components can be separated using low-pass and high-pass filters, respectively. The sensor response to temperature can be extracted from the cavities' component using a correlation method. The device achieved a temperature sensitivity of $-10.8 \pm 0.2 \mathrm{pm} /{ }^{\circ} \mathrm{C}$ between $30{ }^{\circ} \mathrm{C}$ and $80{ }^{\circ} \mathrm{C}$. The same effect is impossible to be obtained in a normal uncleaved microsphere. The random interferometric component did not provide any information on temperature using the same analysis. However, when changing the temperature, a new and completely distinct reflection spectrum with no apparent correlation with others at different temperatures was achieved.
\end{abstract}

Index Terms-Optical fiber sensor, microsphere, focused ion beam, random interference, temperature measurement, correlation.

\section{INTRODUCTION}

$\mathbf{M}$ ICROSPHERES embedded in optical fiber-based configurations have attracted much attention over the last two decades. They have been explored not only as a micro-resonator to excite whispering gallery modes [1], but also as light focusing devices [2] or for laser applications [3]. Recently, a Fabry-Perot cavity based on a hollow core microsphere was used for strain and pressure sensing, with a low temperature sensitivity of $1.17 \mathrm{pm} /{ }^{\circ} \mathrm{C}$ [4].

Random signals have also been investigated due to their unique characteristics and huge potential for application in dif-

Manuscript received December 11, 2017; revised February 16, 2018; accepted March 17, 2018. Date of publication March 20, 2018; date of current version April 6, 2018. This work was supported in part by the Project NanoSTIMA-North Portugal Regional Operational Programme under Grant NORTE-01-0145-FEDER-000016 and in part by COST Action MP1401-Advanced fiber laser and coherent source as tools for society, manufacturing, and lifescience. The work of A. D. Gomes was supported by FCT under Grant SFRH/BD/129428/2017. (Corresponding author: A. D. Gomes.)

A. D. Gomes and B. Silveira are with INESC TEC, 4150-179 Porto, Portugal, also with the Faculty of Sciences, University of Porto, 4169-007 Porto, Portugal, and also with the Leibniz Institute of Photonic Technology, 07745 Jena, Germany (e-mail: ardcgomes@gmail.com; beatriz.m.silveira@ inesctec.pt).

J. Dellith, M. Becker, M. Rothhard, and H. Bartelt are with the Leibniz Institute of Photonic Technology, 07745 Jena, Germany (e-mail: jan.dellith@ leibniz-ipht.de; martin.becker@leibniz-ipht.de; manfred.rothhardt@ipht-jena. de; hartmut.bartelt@leibniz-ipht.de).

O. Frazão is with INESC TEC, 4150-179 Porto, Portugal, and also with the Faculty of Sciences, University of Porto, 4169-007 Porto, Portugal (e-mail: ofrazao@inesctec.pt).

Color versions of one or more of the figures in this letter are available online at http://ieeexplore.ieee.org.

Digital Object Identifier 10.1109/LPT.2018.2817566 ferent fields. For instance, random signals have been studied and applied for secure optical communications [5], random number generation [6], fiber fault detection [7]-[10], or sensing [8], [11]-[13]. In the field of sensing, a random correlation fiber loop ring down was proposed as an improved alternative to the well-known fiber loop ring down technique using optical random signals in the time domain [12]. Random correlation revealed to be a powerful tool to analyze more complicated systems where traditional interrogation techniques cannot be applied. For example, a cross-correlation method applied to a random reference signal and a probe signal allowed to interrogate a large network of chirped fiber Bragg gratings for strain sensing and also to obtain the precise location of each sensor [8]. The same technique was employed to analyze weak fiber Bragg gratings by using a chaotic fiber laser as an optical source [14]. The correlation method can as well be implemented with random signals in the spectral domain. One example of such application is a random fiber Bragg grating interrogated using a spectrum correlation analysis [13]. The method allowed to obtain multiparameter sensing with this structure. Moreover, a similar device with random spectrum can be used to construct a random fiber laser [15].

In this letter, a new sensing structure based on a cleaved silica microsphere is proposed for temperature sensing. Focused ion beam milling is used to create asymmetry in the microsphere by cleaving it with a certain angle. The produced structure presents not only new cavities, but also random interferometric reflections. A spectral correlation method is adopted to extract the sensor response to temperature.

\section{Sensing Structure And Results}

The microsphere was created at the end of a cleaved single mode fiber using a fusion splicer Sumitomo Electronics (TYPE-71C) in manual mode. The electric arc was centered on the fiber, away from the fiber end. The following parameters were used in the process: $0 \mathrm{~ms}$ pre-fusion time, $2000 \mathrm{~ms}$ fusion time, standard arc power +100 (absolute arc-power was not possible to attain, only relative values). Four electric arcs were applied, creating a microsphere with a diameter of around $322 \mu \mathrm{m}$. A scanning electron microscope image of the microsphere is shown in Figure 1(a).

A Tescan (Lyra XMU) FIB-SEM (focused ion beam scanning electron microscope) was used to cut the top of the microsphere with an angle of 21 degrees. A $50 \mathrm{~nm}$-thick tantalum film was deposited over the structure using a sputtering chamber in order to eliminate charging effects during the focused ion beam milling. The cut was performed using an ion 


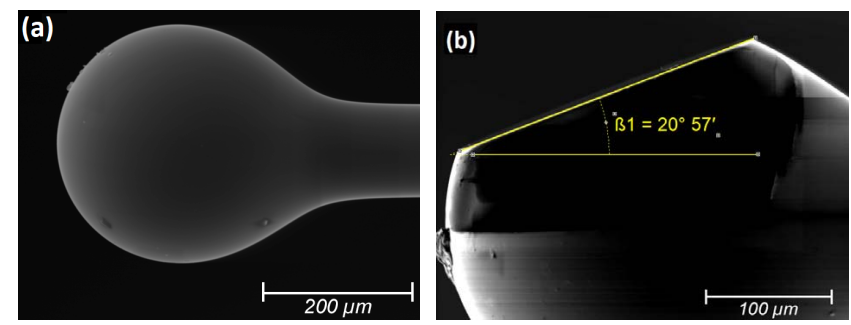

Fig. 1. (a) Scanning electron microscope image of the microsphere. (b) Focused ion beam image of the cleaved microsphere (cutting angle of 21 degrees).

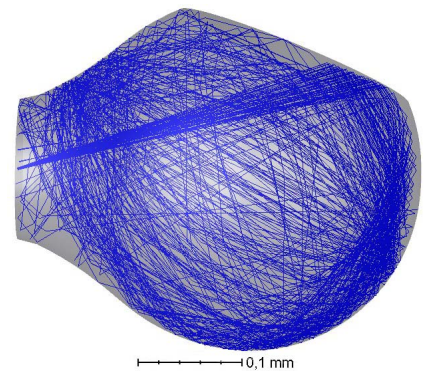

Fig. 2. Random paths of 50 optical rays in the cleaved microsphere modeled using Zemax.

beam current of $10 \mathrm{nA}$. Then, the cleaved surface was polished using an ion beam current of $100 \mathrm{pA}$. A focused ion beam image of the cleaved microsphere is presented in Figure 1(b).

In a conventional silica microsphere, as the one presented in Figure 1(a), light is transmitted to the outwards and a small ercentage is reflected at the microsphere interface. However, after creating asymmetry in the structure by cutting the microsphere, part of the light is reflected in different directions originating from random reflections inside the microsphere that will interfere when collected back into the fiber.

In order to demonstrate the presence of random interferometric reflections inside of the cleaved microsphere, the path of 50 optical rays randomly distributed within a cone of 5 degrees of aperture was simulated using Zemax OpticStudio 16.5. The result is shown in Figure 2. Light collected back into the fiber from different directions will have different phases since they travelled through different random paths. Therefore, the reflection spectrum presents a random-like interferometric behavior.

The reflection spectra of a conventional and a cleaved silica microsphere are depicted in Figure 3. A broadband optical source, an optical circulator, and an optical spectrum analyzer (OSA) with a resolution of $20 \mathrm{pm}$ were used to obtain the reflection spectra. The optical source had a central wavelength of $1550 \mathrm{~nm}$ and a bandwidth of $100 \mathrm{~nm}$. The reflected signal was previously normalized, taking as a reference the reflected signal from a cleaved single mode fiber in air (4\% Fresnel reflection).

The normal uncleaved silica microsphere shows some losses because only part of the $4 \%$ reflected light is collected back into the fiber. It is not possible to perform temperature measurements with this structure since the reflected signal does not present any interferometric spectral modulation behavior,

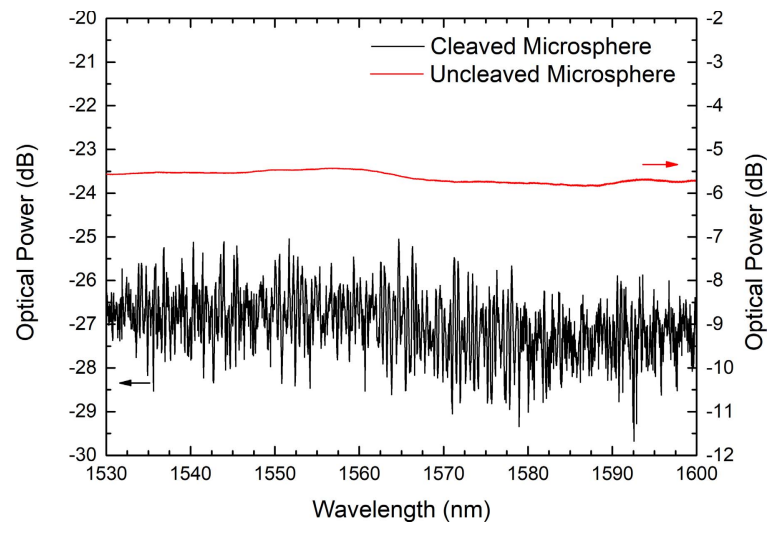

Fig. 3. Reflection spectra of a conventional and a cleaved silica microsphere.

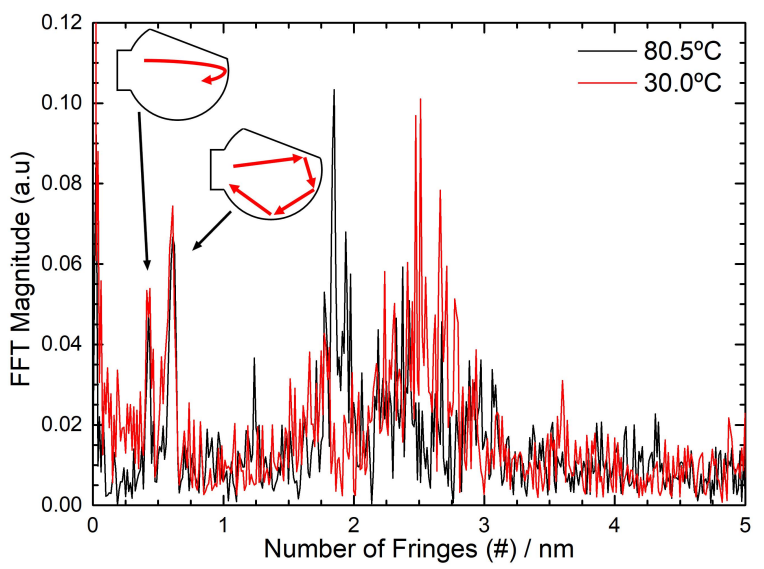

Fig. 4. Fast Fourier transform of the transmission spectrum at two different temperatures.

as one can see from Figure 3. Therefore, conventional peak wavelength-shift inspection methods cannot be used. However, after cleaving the microsphere, the reflected signal presents a random-like interferometric behavior with strong spectral modulation of around $3 \mathrm{~dB}$ amplitude. Note that the reflected signal intensity in the cleaved microsphere is lower due to the presence of some tantalum coating which absorbs part of the light.

A fast Fourier transform (FFT) was applied to the cleaved microsphere reflection spectrum. The result is depicted in Figure 4 for 2 distinct temperatures. The FFT exhibits two different regions: a region with two peaks at low frequency corresponding to cavities in the structure, and a region presenting a band of higher frequencies corresponding to the random interferometric reflections. The two peaks at low frequency $(0.425 \# / \mathrm{nm}$ and $0.612 \# / \mathrm{nm})$ result from two cavities with cavity lengths of around $360 \mu \mathrm{m}$ and $520 \mu \mathrm{m}$, respectively. The estimate value for the cavity length was calculated from the equation for a Fabry-Perot cavity [16]:

$$
L=\frac{\lambda_{1} \lambda_{2}}{2 \times F S R \times n}=\frac{\lambda_{1} \lambda_{2} \times \text { frequency }}{2 n},
$$

where $F S R$ is the free spectral range, $n$ is the refractive index of silica (1.444), the frequency is the inverse of FSR, obtained in the FFT. To calculate the estimate values for 


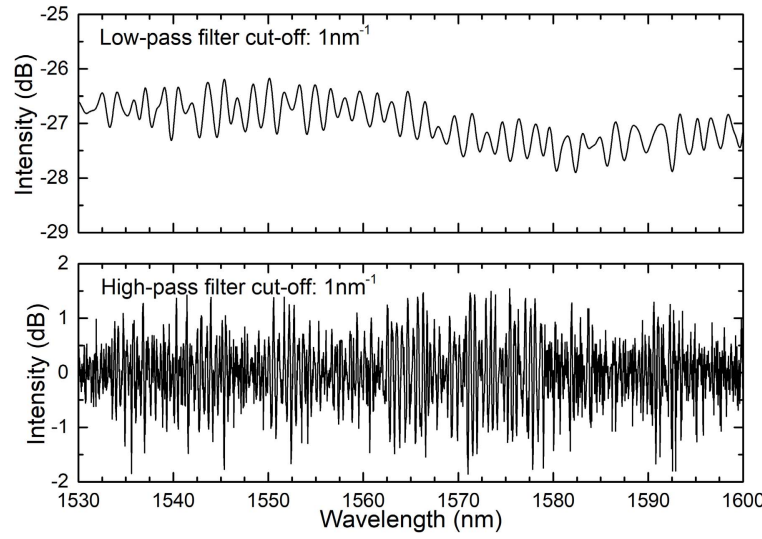

Fig. 5. Filtered spectrum at $80^{\circ} \mathrm{C}$. Upper spectrum: low-pass filter applied to extract the interference between the two cavities. Lower spectrum: high-pass filter applied to extract the random interferometric component.

the cavity lengths, $\lambda_{1}$ and $\lambda_{2}$ were considered $1530 \mathrm{~nm}$ and $1600 \mathrm{~nm}$. Such cavities coincide with the reflection on the microsphere edge and with a reflection on the cleaved region, promoting part of the light to travel around the microsphere before being collected back into the fiber, as displayed in the schematics of Figure 4. The thermal expansion introduces a small change in the cavities length, however in terms of frequency this change is very small and not visible in the FFT of Figure 4. The band of higher frequencies is a consequence of the interference between multiple random reflections inside the microsphere, which present different optical paths between them. Thus, the thermal expansion caused by temperature variation induces a change in these optical paths, causing the interferometric signal to change randomly, producing a different band of frequencies with no apparent relation to the previous one at a different temperature. In Figure 4 the difference between the bands of higher frequencies at different temperatures is clearly visible.

The two regions of the reflection spectrum were separated using a low-pass and high-pass filter, respectively, with cutoff frequency of $1 \# / \mathrm{nm}$. The filtered components are depicted in Figure 5. The upper spectrum corresponds to the two cavities pointed in Figure 4, and the lower spectrum corresponds to the random interferometric part.

The structure was submitted to different temperatures, in air, decreasing from $80.5{ }^{\circ} \mathrm{C}$ until $30{ }^{\circ} \mathrm{C}$. The two components of the reflection spectra were extracted in the same way as in Figure 5. Regarding the two cavities' component, due to the presence of two interferences it is impossible to analyze the spectral response of the sensor at different temperatures by conventional peak wavelength-shift inspection methods. Instead, a spectral cross-correlation analysis was used to obtain the spectral shift information. The cross-correlation between two reflection spectra is calculated using the equation [13]:

$$
R_{x y}(n)=\frac{1}{N} \sum_{k=0}^{N-1} x(k) \cdot y(n+k),
$$

where $\mathrm{N}$ is the total sampling data points, $\mathrm{x}(\mathrm{n})$ and $\mathrm{y}(\mathrm{n})$ are the two spectral data, and $\mathrm{n}$ is a value between $-(\mathrm{N}-1)$ and $\mathrm{N}-1$.

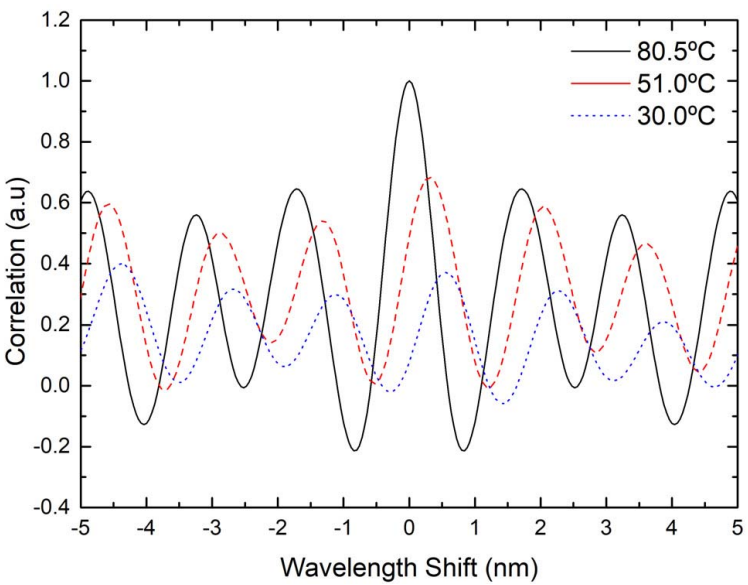

Fig. 6. Normalized autocorrelation (at $80.5^{\circ} \mathrm{C}$ ) and cross-correlation of the reflection spectrum at two distinct temperature $\left(51{ }^{\circ} \mathrm{C}\right.$ and $\left.30{ }^{\circ} \mathrm{C}\right)$.

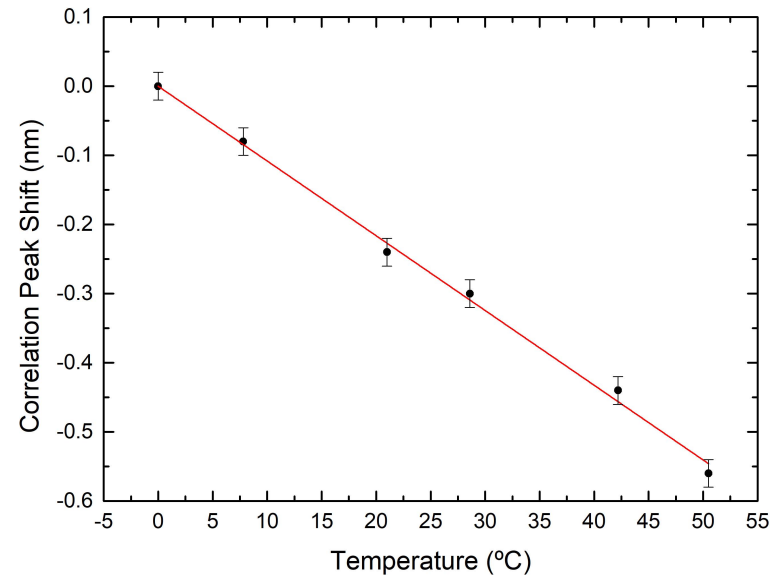

Fig. 7. Correlation wavelength shift, around $0 \mathrm{~nm}$, as a function of temperature variation.

Elements of $x(n)$ and $y(n)$ with negative indices or larger than $\mathrm{N}-1$ are assumed as zero. If $\mathrm{x}(\mathrm{n})$ and $\mathrm{y}(\mathrm{n})$ are the same signal, the operation is called autocorrelation.

To better obtain the correlation spectrum, the DC component (around $27 \mathrm{~dB}$ ) was removed in all the reflection spectra, centering them at $0 \mathrm{~dB}$. The normalized autocorrelation and cross-correlation of the reflection spectra for two distinct temperatures are depicted in Figure 6. The multiple peaks observed in the autocorrelation and cross-correlations means that the signal is periodic. It is visible that the correlation peaks shift when exposing the sensor to a different temperature. In this case, the reflection spectrum at $80.5{ }^{\circ} \mathrm{C}$ was used as a reference and the autocorrelation was calculated. Then, the cross-correlation was performed between the reflection spectra at different temperatures and the reference one. The correlation peak wavelength shift around $0 \mathrm{~nm}$ was monitored as a function of temperature variation. The result is shown in Figure 7. The sensor achieved a temperature sensitivity of $-10.8 \pm 0.2 \mathrm{pm} /{ }^{\circ} \mathrm{C}$ between $80.5{ }^{\circ} \mathrm{C}$ and $30{ }^{\circ} \mathrm{C}$. This value of sensitivity is an average of the temperature sensitivity across the considered wavelength range. To have a more restricted value of sensitivity one can also perform 
the correlation analysis in different wavelength regions (shorter wavelength, higher wavelength) and restrict the correlation analysis to that wavelength region, since the sensitivity value should depend on the wavelength [13].

Regarding the random interferometric component, no temperature information can be obtained through the spectrum. Using the same correlation analysis, there are no correlation peaks that can be monitored to extract the temperature response. As discussed previously, this band of frequencies changes randomly with temperature, therefore producing a new and completely different noisy spectrum with no apparent relation with others at different temperature.

\section{CONCLUSIONS}

In conclusion, a novel structure based on a cleaved silica microsphere on top of a fiber tip was developed and proposed for temperature sensing. With a normal uncleaved silica microsphere, it is impossible to obtain an interferometric behavior that allows to extract a response to temperature. However, when introducing asymmetry in the structure by focused ion beam cleaving the microsphere, not only new cavities are formed but also random interferometric reflections are promoted inside the microsphere, originating a random-like reflection spectrum. Two distinct regions can be observed when a FFT is applied. The first involves two cavities at a lower frequency formed by the reflection on the microsphere edge and a reflection on the cut that guides light through successive reflections across the bottom border of the microsphere. The second region involves a band of frequencies that is originated by the random interferometric reflections. These two spectral characteristics can be separated using low-pass and high-pass filters, respectively. The sensor response to temperature can be extracted from the two-cavity component using a correlation method. The device achieved a temperature sensitivity of $-10.8 \pm 0.2 \mathrm{pm} /{ }^{\circ} \mathrm{C}$ between $80.5^{\circ} \mathrm{C}$ and $30{ }^{\circ} \mathrm{C}$, which is similar to a fiber Bragg grating [17]. The random interferometric component did not provide any information on temperature using the same analysis. This is in fact different from the random fiber Bragg grating proposed by $\mathrm{Xu}$ et al. [13], where the obtained random spectra at different temperatures are correlated, and therefore a crosscorrelation method can extract a temperature response. In the case of the proposed cleaved microsphere, no correlation was found between the different spectra at different temperatures. The existence of the cut in the structure is crucial to create asymmetry and introduce interferometric information in the spectrum that allows to extract a temperature response. Instead of using a focused ion beam to cleave the microsphere, which is an expensive technique, a similar cut can be obtained by performing a controlled polishing of the microsphere. In the future, a study should be done to find the best cutting angle in order to obtain more random reflections inside the microsphere. Such cut would provide more spectral components to the reflection spectrum.

\section{REFERENCES}

[1] J. C. Knight, G. Cheung, F. Jacques, and T. A. Birks, "Phase-matched excitation of whispering-gallery-mode resonances by a fiber taper," Opt. Lett., vol. 22, no. 15, pp. 1129-1131, 1997.

[2] A. M. Kapitonov and V. N. Astratov, "Observation of nanojet-induced modes with small propagation losses in chains of coupled spherical cavities," Opt. Lett., vol. 32, no. 4, pp. 409-411, 2007.

[3] F. Lissillour, D. Messager, G. Stéphan, and P. Féron, "Whisperinggallery-mode laser at $1.56 \mu \mathrm{m}$ excited by a fiber taper," Opt. Lett., vol. 26, no. 14, pp. 1051-1053, 2001.

[4] C. Monteiro, S. Silva, and O. Frazão, "Hollow microsphere Fabry-Pérot cavity for sensing applications," IEEE Photon. Technol. Lett., vol. 29, no. 15, pp. 1229-1232, Aug. 1, 2017.

[5] A. Argyris et al., "Chaos-based communications at high bit rates using commercial fibre-optic links," Nature, vol. 438, no. 7066, pp. 343-346, Nov. 2005.

[6] A. Uchida et al., "Fast physical random bit generation with chaotic semiconductor lasers," Nature Photon., vol. 2, no. 12, pp. 728-732, Dec. 2008.

[7] Y. Wang, B. Wang, and A. Wang, "Chaotic correlation optical time domain reflectometer utilizing laser diode," IEEE Photon. Technol. Lett., vol. 20, no. 19, pp. 1636-1638, Oct. 1, 2008.

[8] Y. Luo et al., "Optical chaos and hybrid WDM/TDM based large capacity quasi-distributed sensing network with real-time fiber fault monitoring," Opt. Exp., vol. 23, no. 3, pp. 2416-2423, 2015.

[9] L. Xia, D. Huang, J. Xu, and D. Liu, "Simultaneous and precise fault locating in WDM-PON by the generation of optical wideband chaos," Opt. Lett., vol. 38, no. 19, pp. 3762-3764, 2013.

[10] Z. N. Wang et al., "Long-range and high-precision correlation optical time-domain reflectometry utilizing an all-fiber chaotic source," Opt. Exp., vol. 23, no. 12, pp. 15514-15520, 2015.

[11] D. Rontani, D. Choi, C.-Y. Chang, A. Locquet, and D. S. Citrin, "Compressive sensing with optical chaos," Sci. Rep., vol. 6, Dec. 2016, Art. no. 35206.

[12] L. Yang et al., "Optical sensors using chaotic correlation fiber loop ring down," Opt. Exp., vol. 25, no. 3, pp. 2031-2037, 2017.

[13] Y. Xu et al., "Optical fiber random grating-based multiparameter sensor," Opt. Lett., vol. 40, no. 23, pp. 5514-5517, 2015.

[14] J. Zhang, L. Yang, H. Yang, L. Zhang, J. Wang, and Z. Zhang, "A novel demodulation scheme for high precision quasi-distributed sensing system based on chaotic fiber laser," Sens. Actuators A, Phys., vol. 233, pp. 427-433, Sep. 2015.

[15] Y. Xu, M. Zhang, P. Lu, S. Mihailov, X. Bao, and C. Canada, "Multiparameter fiber-optic sensors based on fiber random grating," Proc. SPIE, vol. 10323, pp. 1032308-1-1032308-4, Apr. 2017.

[16] C. S. Monteiro et al., "Fiber Fabry-Pérot interferometer for curvature sensing," Photon. Sensors, vol. 6, no. 4, pp. 339-344, 2016.

[17] K. O. Hill and G. Meltz, "Fiber Bragg grating technology fundamentals and overview," J. Lightw. Technol., vol. 15, no. 8, pp. 1263-1276, 1997. 